\title{
ON THE SINGULARITIES OF NONLINEAR FREDHOLM OPERATORS OF POSITIVE INDEX
}

\author{
M. S. BERGER ${ }^{1}$ AND R. A. PLASTOCK
}

\begin{abstract}
The singular set $B=\left\{x \mid F^{\prime}(x)\right.$ is not surjective $\}$ of a nonlinear Fredholm operator $F$ of positive index (between Banach spaces $X_{1}$ and $X_{2}$ ) is investigated. Under the assumption that the mapping is proper and has a locally Lipschitzian Fréchet derivative $F^{\prime}(x)$, it is shown that the singular set $B$ is nonempty. Furthermore, when the Banach spaces are infinite dimensional, $B$ cannot be the countable union of compact sets nor can $F^{-1}(F(B))$ contain isolated points.
\end{abstract}

Since the original paper of Smale [1], much attention has focused on the properties of nonlinear Fredholm operators of positive index. These operators are currently of enhanced interest because of their connection with the self-dual solutions of the nonlinear Euclidean Yang-Mills equations [2]. Recall that $C^{1}$ mapping $F$ between two Banach spaces $X_{1}$ and $X_{2}$ is called Fredholm if its Fréchet derivative $F^{\prime}(x)$ is a linear Fredholm operator for each $x \in X_{1}$. The index of $F=$ index $F^{\prime}(x)$ and since $F^{\prime}(x)$ depends continuously on $x$, this index does not depend on $x \in X_{1}$. In this article we wish to point out some interesting properties of the set of singular points of $F$ (i.e., the set of points $x \in X_{1}$ such that $F^{\prime}(x)$ is not surjective). In particular, we wish to show that new phenomena occur for the study of singular points of proper operators $F$ of strictly positive index that have no analogue in the index zero case (cf. [3]). Throughout this paper we make the standing assumption that the Fréchet derivative $F^{\prime}(x)$ of $F(x)$ is locally Lipschitz.

A novelty of our results (Theorems 3 and 4 ) is that they apply only to mappings between infinite-dimensional Banach spaces. We shall explore the implications of our results for nonlinear elliptic boundary value problems in a later paper. The authors are grateful to the referee for a helpful simplification and for pointing out an error in an earlier version.

Some basic facts. A key difference between the study of nonlinear Fredholm operators of strictly positive index and operators of index zero is the use of the higher homotopy groups and fibre bundle arguments instead of the fundamental group and covering space arguments.

Received by the editors January 18, 1979 and, in revised form, April 3, 1979, April 4, 1979 and June 5, 1979.

AMS (MOS) subject classifications (1970). Primary 47C15, 46N05; Secondary 35H60, $57 \mathrm{~B} 45$.

Key words and phrases. Nonlinear Fredholm operator, fiber bundle map, higher homotopy groups.

${ }^{1}$ Research partially supported by an NSF grant. 
Definition. A mapping $F: E \rightarrow B$ is said to be a fibre bundle map or fibration if there is an open covering $\left\{U_{\alpha}\right\}$ of $B$, a space $M$ (called the fibre) and for each $U_{\alpha}$ a homeomorphism $\phi_{\alpha}: F^{-1}\left(U_{\alpha}\right) \rightarrow U_{\alpha} \times M$ such that $F=P \phi_{\alpha}$ where $P$ is the natural projection $B \times M \rightarrow B$.

(For the study of Fredholm operators of positive index, it is necessary to study fibres $M$ that are not discrete.) Key facts about fibre bundle maps that we shall use are:

(A) Let $F: E \rightarrow B$ be a fibre bundle map. Let $M=F^{-1}\left(b_{0}\right)$ and choose $e_{0} \in M$. Then the following relations between higher homotopy groups hold:

(i) The homotopy group sequence

$$
\begin{aligned}
\rightarrow \pi_{n+1}\left(B, b_{0}\right) \rightarrow \pi_{n}\left(M, e_{0}\right) \rightarrow & \pi_{n}\left(E, e_{0}\right) \rightarrow \cdots \rightarrow \pi_{1}\left(B, b_{0}\right) \\
& \rightarrow \cdots \rightarrow \pi_{0}\left(M, e_{0}\right) \rightarrow \pi_{0}\left(E, e_{0}\right) \rightarrow \pi_{0}\left(B, b_{0}\right)
\end{aligned}
$$

is exact.

(ii) If $M$ is contractible in $E$, then for $n \geqslant 2$,

$$
\pi_{n}\left(B, b_{0}\right) \cong \pi_{n}\left(E, e_{0}\right) \oplus \pi_{n-1}\left(M, e_{0}\right)
$$

(B) Let $U$ be an open connected set in a Banach space $X_{1}$ and $F: U \rightarrow F(U)$ be a proper $C^{\prime}$ Fredholm map of index $p>0$, whose Fréchet derivative satisfies a local Lipschitz condition. If the singular point set $B$ of $F$ is vacuous, then $F$ is a fibre bundle map and the fibres $F^{-1}(y)$ are homeomorphic compact $p$-dimensional manifolds.

Proofs can be found in [5] and [6].

Results on singular points. We begin by proving a result that contrasts sharply with the case of index 0 , namely, the well-known result (see [4, p. 514]) that a proper local homeomorphism between two Banach spaces is a global homeomorphism.

Theorem 1. Let $F: X \rightarrow Y$ be a proper Fredholm operator of index $p>0$. Then $F$ must have singular points.

Proof. We suppose the set of singular points $B$ of $F$ is empty, and obtain a contradiction. Indeed, if $B=\varnothing$, the empty set, $F$ is surjective, so that $F(X)=Y$ since $F(X)$ is both open and closed in $Y$, and so the result (B) on fibre bundle maps implies $F$ is a fibre bundle mapping between the Banach spaces $X$ and $Y$. Now we apply the result (A)(i) on fibre bundle maps to this case. Here $\pi_{i}(X)=\pi_{i}(Y)=0$ for all $i \geqslant 0$ since $X$ and $Y$ are contractible. Consequently, the exactness of the homotopy sequence with $M=F^{-1}(y)$ for any point $y \in Y$ implies $\pi_{i}\left(F^{-1}(y)\right)=0$ for all $i \geqslant 0$. Hence $F^{-1}(y)$ is contractible, and also by [1] a compact $p$-dimensional manifold without boundary. This is the desired contradiction, since such compact $p$-manifolds possess a mod 2 degree as in [8], defined for $C^{\prime}$ self-mappings with different degrees for the identity and constant maps.

We now investigate the behaviour of $F$ off the singular points of $F$. 
TheORem 2. Let $S=F(B)$ denote the set of singular values for any nonlinear Fredholm operator $F$. Suppose $F$ is a proper Fredholm operator acting between the Banach spaces $X$ and $Y$. Then $Y-S$ is the union of path components such that the sets, $F^{-1}(y)$ are homeomorphic compact p-dimensional manifolds (possibly empty) as $y$ varies in each such path component. Furthermore, assuming $X \neq F^{-1}(S), X-$ $F^{-1}(S)$ is the union of path components $\left\{C_{\alpha}\right\}$ each of which is mapped onto a path component of $Y-S$ and such that $\left\{F^{-1}(y) \cap C_{\alpha}\right\}$ are homeomorphic compact p-dimensional manifolds as $y$ varies in each path component of $Y-S$.

Proof. Writing $\tilde{B}=F^{-1}(S)$, we first show that $F: X-\tilde{B} \rightarrow Y-S$ is a proper map between these two sets. For if $K \subset Y-S$ is compact, it is certainly a compact set in $Y$. Thus $F^{-1}(K)$ is compact in $X$, and again it is contained in $X-\tilde{B}$ and so is compact there, as was to be shown.

Since $X-\tilde{B}$ is open and contains no singular points, $F$ is an open map on it, and so $F[X-\tilde{B}]$ is an open and closed (by properness) subset of $Y-S$ and must be the union of path components of $Y-S$. By arguing as above, $F: X-\tilde{B} \rightarrow F[X$ $-\tilde{B}]$ is also a proper map, and applying (B) of $\S 1$, we conclude that it is in fact a fibration. Now if $Y_{\alpha}$ is a path component of $Y-S$, then either $Y_{\alpha} \subset F[X-\tilde{B}]$ or $Y_{\alpha} \cap F[X-\tilde{B}]=\varnothing$. In the latter case, if $y \in Y_{\alpha}$, obviously $F^{-1}(y)=\varnothing$. In the former case, by the properties of fibrations [6, p. 71], $F: F^{-1}\left[Y_{\alpha}\right] \rightarrow Y_{\alpha}$ is a fibration, and so $F^{-1}(y)$ are homeomorphic, compact $p$-manifolds. Also, if $C_{\alpha}$ is a path component of $X-B$, it follows [6, p. 71] that $F$ is a fibration of $C_{\alpha}$ onto some path component $Y_{\alpha}$ of $Y-S$, and so $F^{-1}(y) \cap C_{\alpha}$ are homeomorphic for $y \in Y_{\beta}$.

Since every proper nonlinear Fredholm operator of strictly positive index must possess singular points, it is natural to inquire about the structure of such singular points. For mappings between infinite-dimensional Banach spaces, the following two results hold.

THEOREM 3. Let $F$ be a proper nonlinear Fredholm operator of index $p>0$, between Banach spaces of infinite dimension. Then $\tilde{B}=F^{-1}(S)$ has no isolated points.

Proof. We argue by contradiction, supposing $b \in F^{-1}(S)$ is an isolated point of $F^{-1}(S)$. Let $g=F(b)$. First we find an open ball $O$, about $g$, so small that the connected component $C$ of $F^{-1}(O)$ which contains $b$ satisfies $C \cap \tilde{B}=b$.

To find the open ball $O$, we proceed as follows. Choose an open ball $A$ about $b$ so that $\bar{A} \cap \tilde{B}=b$. Let $F(b)=g$. Then $F^{-1}(g) \cap \partial A=\varnothing$ and $\tilde{B} \cap A=b$.

About $g$ choose open balls $O_{n}$ of radius $1 / n$. Let $C_{n}$ be the component of $F^{-1}\left(O_{n}\right) \cap A$ containing $b$. For some $N, \bar{C}_{N} \cap \partial A=\varnothing$. (If not, then we can find a sequence $x_{n} \in C_{n} \cap \partial A$. Then $F\left(x_{n}\right) \rightarrow g$, and by the properness of $F$, a subsequence of the $x_{n}$ converges to some $\bar{x} \in F^{-1}(g) \cap \partial A$, contradicting our construction of $A$.) Thus $\bar{C}_{N} \subset A$ by connectedness. Hence $C_{N} \subset A$. Setting $C=C_{N}$ and $O=O_{N}$ we have $C \cap \tilde{B}=b$ and the desired open ball.

We now show that $F: C-b \rightarrow O-g$ is a proper map. Let $K \subset O-g$ be compact. Then $K$ is compact in $Y$ and so $\tilde{K}=F^{-1}(K)$ is compact in $X$. Then $\tilde{K} \cap \bar{C}$ is compact. We shall show that $\tilde{K} \cap \bar{C}=\tilde{K} \cap C(=\tilde{K} \cap C-b)$ and 
thus establish the desired properness property. Indeed, if $x \in \tilde{K} \cap \bar{C}$, then $x$ lies in some component $\tilde{C}$ of $F^{-1}(O)$. Since these components are open sets, $x$ is an interior point of $\tilde{C}$. It is also a boundary point of $C$. Thus every open neighborhood of $x$ contains points of $C$ which also belong to $\tilde{C}$. Thus $\tilde{C}=C$ since both are components, and so $x \in \tilde{K} \cap C$ as we wanted.

Thus the fact that $C$ is a component and an open set yields the fact that $F$ is a proper map of $C-b$ onto $O-g$. Furthermore $C-b$ contains no singular points and is open and connected. Applying (B) of $\S 1$ we conclude that $F: C-b \rightarrow O-$ $g$ is a fiber bundle map.

Let $\bar{y} \in O-g$. We now show that the fiber $\bar{F}=F^{-1}(\bar{y}) \cap C-b$ is contractible in $C-b$. For the path $L(t)=(1-t) g+t \bar{y}, t>0$, there is a lifting function $H(\bar{x}, t)(\bar{x} \in \bar{F}, t>0)$ in $C-b$ so that $H(\bar{x}, 1)=\bar{x}$ and $F(H(\bar{x}, t))=L(t)$. (Spanier [6, p. 92, Theorem 8].) By the properness of $F$ and the fact that $b$ is also an isolated point of $F^{-1}(g), H(\bar{x}, 0)=b$. Thus $H(\bar{x}, t)$ defines a homotopy of the identity map on the fiber $\bar{F}$ and the point $b$. Thus $\bar{F}$ is contractible in $C$ and so is also contractible in $C-b$ due to the infinite dimensionality of our spaces [7].

We now obtain a contradiction: Since $Y$ is infinite dimensional, by [7]

$$
\pi_{i}(O-g)=0 \quad \forall i>0
$$

and moreover $C-b$ is connected. We apply the exact homotopy sequence of (A)(i) of $\$ 1$ to conclude that

$$
\pi_{i}(C-b) \simeq \pi_{i}(\bar{F})
$$

Since $\bar{F}$ is contractible in $C-b$, we apply (A)(ii) and conclude that for $i>2$,

$$
\pi_{i}(O-g) \simeq \pi_{i}(C-b)+\pi_{i-1}(\bar{F}) .
$$

Since the left-hand side is zero, we conclude from $(*)$ that

$$
\pi_{i-1}(\bar{F})=0 \text { for } i>2 \text {. }
$$

Using (*) again we see that $\pi_{0}(\bar{F})=0$ and so

$$
\pi_{i}(\bar{F})=0, \quad i>0 .
$$

Again $\bar{F}$ is contractible, and as in the proof of Theorem 1 we obtain a contradiction since $\bar{F}$ is a compact orientable and connected $p$-dimensional manifold, without boundary.

This result is a purely infinite-dimensional one. In fact, Timourian has shown that in the finite-dimensional case $F: M^{n} \rightarrow N^{p}$ ( $n$ - and $p$-dimensional manifolds, respectively) where $F$ is $C^{\prime}$, proper with index $n-p>0$ that an isolated point of $F^{-1}(S)$ can exist only in the cases $(n, p)=(n, 1),(4,3),(8,5)$ or $(16,9)$ !.

Our final result shows that, in the infinite-dimensional case, the singular set $B$ cannot be the countable union of compact sets.

Theorem 4. Let $F: X \rightarrow Y$ be a $C^{\prime}$ proper Fredholm map with index $F>0$. Then the singular set $B$ cannot be the countable union of compact sets.

Proof. Let $B$ be the countable union of compact sets. Since $B$ is closed, so is $S=F(B)$ and also $F^{-1}(S)$ by the properness of $F$. Thus $X-F^{-1}(S)$ and $Y-S$ 
are both open. Since both $S$ and $F^{-1}(S)$ are also the countable union of compact sets, $X-F^{-1}(S)$ and $Y-S$ are homeomorphic to $X$ and $Y$, respectively [7]. In particular, both are connected and contractible. We shall derive a contradiction by showing that $F$ is a fibre bundle map of $X-F^{-1}(S)$ onto $Y-S$. Once this is done we argue, using the contractibility of both spaces, as in Theorem 1 to conclude the existence of a singular point of $F$ in $X-F^{-1}(S)$-a contradiction since this set contains no singular points.

To proceed, we argue exactly as in the beginning of Theorem 1 to conclude that $F$ is a proper map on $X-F^{-1}(S)$ to $Y-S$. Since $X-F^{-1}(S)$ contains no singular points, $F$ is an open map on this set and so $F\left[X-F^{-1}(S)\right]$ is an open and closed subset of the connected set $Y-S$. Hence $F$ maps $X-F^{-1}(S)$ onto $Y-S$. We now apply (B) of $\S 1$ to derive that $F$ is a fibre bundle map and so the proof is complete.

\section{BIBLIOGRAPHY}

1. S. Smale, An infinite dimensional version of Sard's theorem, Amer. J. Math. 87 (1965), 861-867.

2. M. Atiyah, N. Hitchin and I. Singer, Self-duality in four-dimensional Riemannian geometry, Proc. Roy. Soc. London Ser. A 362 (1978), 425-461.

3. R. Plastock, Nonlinear Fredholm maps of index zero and their singularities, Proc. Amer. Math. Soc. 68 (1978), 317-322.

4. M. S. Berger, Nonlinearity and functional analysis, Academic Press, New York, 1977.

5. C. Earle and J. Eells, Foliations and fibrations, J. Differential Geometry 7 (1967), 61-69.

6. E. Spanier, Algebraic topology, McGraw-Hill, New York, 1966.

7. W. H. Cutler, Negligible subsets of infinite-dimensional Fréchet spaces, Proc. Amer. Math. Soc. 23 (1969), 668-675.

8. J. W. Milnor, Topology from the differentiable viewpoint, Univ. of Virginia Press, Charlottesville, Virginia, 1965.

Department of Mathematics, The Institute for Advanced Study, Prnnceton, New Jersey 08540

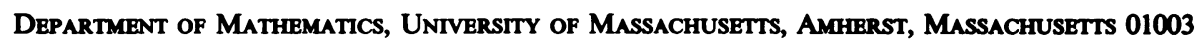

Department of Mathematics, New Jersey Institute of Technology, Newark, New Jersey 07102 